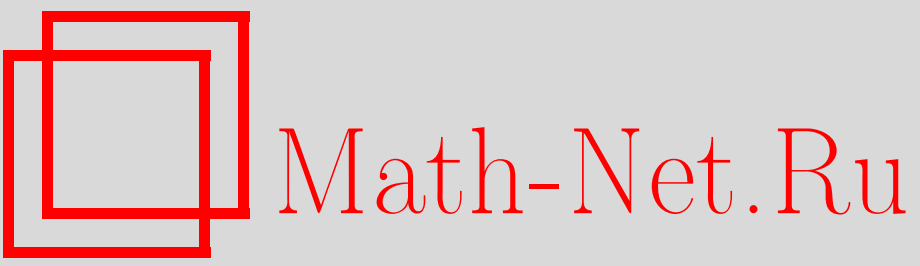

Е. С. Корчак, Анализ математической модели торможения подвижной поперечины гидравлического пресса в верхнем положении, Вестн. Сам. гос. техн. ун-та. Сер. Физ.-мат. науки, 2009, выпуск 2(), 153-159

DOI: https://doi.org/10.14498/vsgtu647

Использование Общероссийского математического портала Math-Net.Ru подразумевает, что вы прочитали и согласны с пользовательским соглашением

http://www.mathnet.ru/rus/agreement

Параметры загрузки:

IP : 54.237 .206 .68

26 апреля 2023 г., $12: 23: 22$ 
УДК 517.958:621.226

\section{АНАЛИЗ МАТЕМАТИЧЕСКОЙ МОДЕЛИ ТОРМОЖЕНИЯ ПОДВИЖНОЙ ПОПЕРЕЧИНЫ ГИДРАВЛИЧЕСКОГО ПРЕССА В ВЕРХНЕМ ПОЛОЖЕНИИ}

\section{E. C. Корчак}

Донбасская государственная машиностроительная академия, Украина, 84313, г. Краматорск, ул. Шкадинова, 72.

E-mail: helen_korchak@ukr.net

Рассмотрена математическая модель торможения подвижной поперечины гидравлического пресса в верхнем положении. Проанализировано влияние времени закрытия регулирующего клапана, его конструктивной характеристики и коэфбиииента качества гидросистемы на динамику торможения поперечины на разных этапах.

Ключевые слова: торможение, подвижная поперечина, гидравлический пресс, регулирующий клапан, конструктивная характеристика, время закрытия.

Введение. При торможении подвижных частей гидравлического пресса гидропривод должен не только поглотить их кинетическую энергию, но и обеспечить оптимальный закон движения, при котором создаются наиболее благоприятные условия для выполнения технологического процесса и обеспечивается наибольшая производительность машины [1]. Для достижения наилучших режимов остановки подвижной поперечины должны быть исключены условия, способствующие возникновению колебаний и ударов, а также динамических перегрузок частей пресса.

1. Механизм процесса торможения подвижной поперечины гидравлического пресса в верхнем положении. Торможение подвижной поперечины в верхнем положении осуществляется путем закрытия впускного клапана возвратных цилиндров, чем достигается снижение активной силы подъёма, и поперечина замедляет свое движение [2]. При торможении [3] приведённая скорость жидкости на первом участке гидропривода «аккумулятор - регулирующий клапан» (рис. 1) под воздействием закрывающегося клапана падает быстрее, чем скорость поперечины и приведённая к ней скорость жидкости на втором участке системы «регулирующий клапан - возвратный цилиндр».

По мере закрытия клапана разница приведённых скоростей жидкости на первом и втором участках гидропривода возрастает и достигает максимума в момент полного закрытия регулирующего клапана, когда поток жидкости на первом участке остановлен и его взаимодействие со вторым участком прекратилось. Таким образом, жидкость в магистрали «аккумулятор - пресс» и металлические подвижные части, двигавшиеся до закрытия клапана как одна масса, приобретают характер двухмассовой системы. При этом разделителем масс служит закрывающийся регулирующий клапан. Поэтому при составлении математической модели торможения подвижных частей одной из масс двухмассовой системы приняли приведённую к поперечине массу жидкости на первом участке гидропривода, а второй - сумму масс металлических по-

Елена Сергеевна Корчак (к.т.н.), старший преподаватель, каф. машин и технологии обработки металлов давлением. 


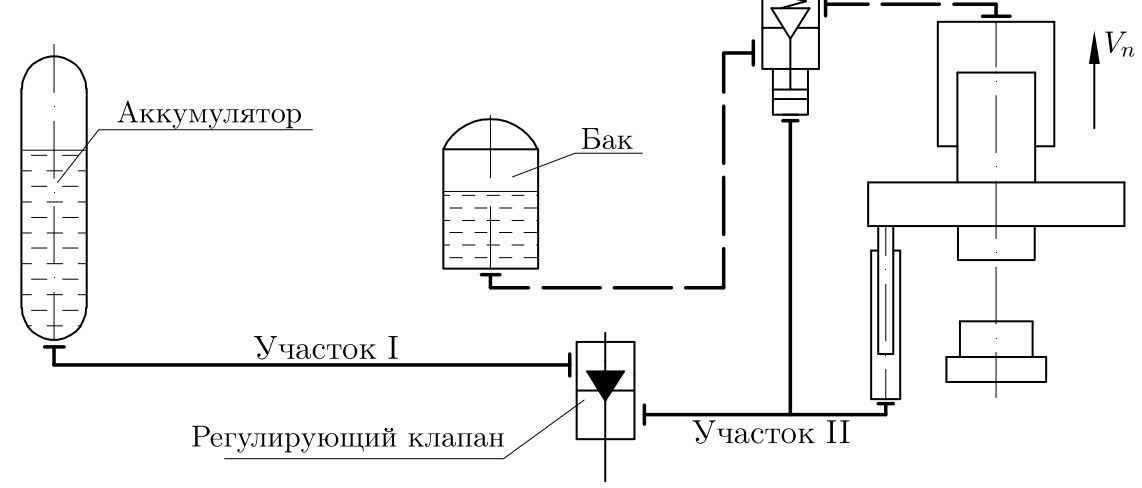

Рис. 1. Схема магистралей «аккумулятор - пресс - бак»

движных частей и жидкости на втором участке гидропривода. Элементом, связывающим обе массы, является невесомая пружина, параметры которой соответствуют приведённой упругости второго участка гидропривода.

Из сказанного выше следует, что анализ процесса торможения подвижной поперечины гидравлического пресса в верхнем положении осуществляется на базе комбинированной «жёстко-упругой» модели гидропривода, в которой жидкость и трубопроводы на первом участке гидропривода считаются «жёсткими», а на втором - «упругими».

2. Описание математической модели. Исследования динамических и силовых особенностей торможения подвижной поперечины показали, что для анализа данной математической модели движение поперечины целесообразно разбить на два этапа, существенно отличающихся динамикой: 1) в процессе закрытия регулирующего клапана; 2) после закрытия регулирующего клапана.

За основу математической модели первого этапа торможения приняли уравнение Рикатти [4], разделив его на два уравнения, соответствующих первому и второму участкам гидропривода и связанных между собой упругой составляющей:

$$
\left\{\begin{array}{l}
a_{1} \frac{d^{2} S_{1}}{d t^{2}}+b\left[1+\alpha\left(\frac{1}{\left(1-\frac{t}{t_{3}}\right)^{2 \cdot n}}-1\right)\right]\left(\frac{d S_{1}}{d t}\right)^{2}-c_{1}-k_{T}\left(S_{2}-S_{1}\right)=0 \\
a_{2} \frac{d^{2} S_{2}}{d t^{2}}-c_{2}+k_{T}\left(S_{2}-S_{1}\right)=0
\end{array}\right.
$$

где $a_{1}, a_{2}$ - приведённые к поперечине движущиеся массы на двух участках гидропривода; $c_{1}, c_{2}$ - активные силы, воздействующие на приведённые массы; $b$ - коэффициент гидравлического сопротивления магистралей; $S_{1}, S_{2}-$ пути, пройденные первой и второй приведёнными массами; $t_{3}-$ время закрытия регулирующего клапана при торможении поперечины; $k_{T}$ - приведённая линейная жёсткость упругих элементов на втором участке гидропривода; $n-$ показатель вида конструктивной характеристики регулирующего клапана; $\alpha$ - коэффициент качества гидросистемы. 
Математическая модель первого этапа торможения соответствует расчётной схеме, приведённой на рис. 2, а.

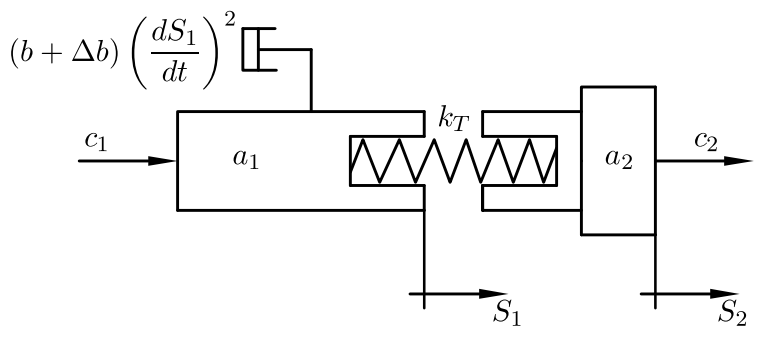

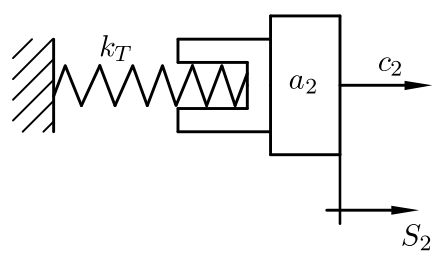

$б$

Рис. 2. Расчётные схемы первого (а) и второго (б) этапов торможения

Член в квадратных скобках первого уравнения системы (1) представляет собой коэффициент, учитывающий прирост гидравлического сопротивления регулирующего клапана в ходе его закрытия. Коэффициент $\alpha$ указывает на степень искажения скоростной характеристики клапана под влиянием гидравлического сопротивления магистрали «аккумулятор - пресс».

На втором этапе торможения в результате закрытия регулирующего клапана происходит разделение участков гидропривода, в результате чего взаимодействие приведённых масс $a_{1}$ и $a_{2}$ прекращается. Масса $a_{1}$ полностью затормаживается клапаном, а масса $a_{2}$ продолжает свое движение.

Взаимодействие сил с подвижной поперечиной на втором этапе торможения иллюстрируется расчётной схемой, приведённой на рис. 2 , б.

За основу описания второго этапа торможения приняли второе уравнение системы (1), описывающее движение приведённой массы $a_{2}$ :

$$
a_{2} \frac{d^{2} S_{2}}{d t^{2}}-c_{2}+k_{T}\left(S_{2}-S_{1 \max }\right)=0,
$$

где $S_{1 \text { max }}$ - путь, пройденный массой $a_{1}$ к моменту закрытия регулирующего клапана.

Система (1) аналитически не решается. Для её решения, а также для решения уравнения (2), использовался численный метод Рунге-Кутта.

Математическая модель торможения подвижной поперечины в верхнем положении содержит ряд параметров $\left(t_{3}, n, \alpha\right)$, оказывающих существенное влияние на динамику остановки поперечины. Проанализируем влияние каждого из них на примере ковочного гидравлического пресса усилием $60 \mathrm{MH}$.

3. Анализ математической модели первого этапа торможения. На рис. 3 представлены результаты моделирования процесса торможения подвижной поперечины на базе системы (1). Впускной клапан возвратных цилиндров с релейной $(\mathrm{a})$, линейной (б) и квадратичной (в) конструктивными характеристиками закрывался за 0,2 с. Коэффициент $\alpha$ принимался равным 0,6.

При торможении клапаном с релейной конструктивной характеристикой (рис. 3, a) резкое снижение скорости $V_{1}$ движения столба жидкости на участке «аккумулятор - регулирующий клапан» наблюдается только в конце закрытия клапана, при этом ускорение $j_{1}$ достигает пикового значения $9 \mathrm{M} / \mathrm{c}^{2}$, что говорит о наличии интенсивного гидроудара в этой магистрали. Скорость $V_{2}$ движения столба жидкости в магистрали «регулирующий клапан - 


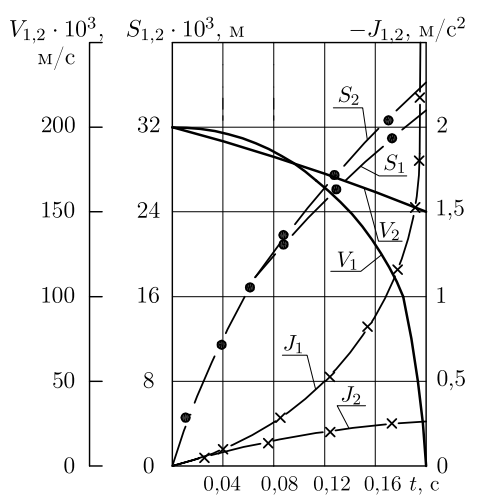

a

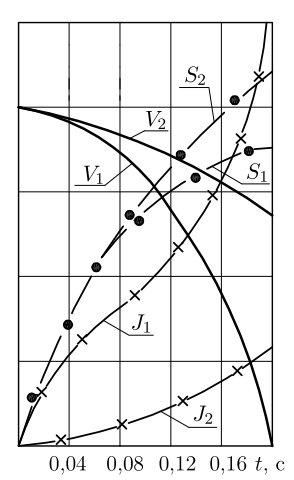

б

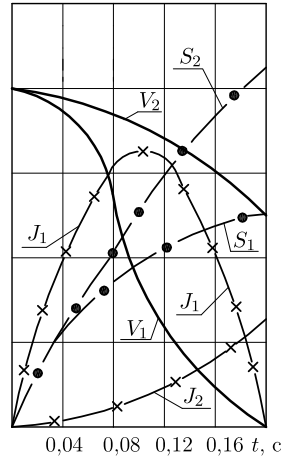

$B$

Рис. 3. Влияние регулирующего клапана на динамику торможения подвижной попере-

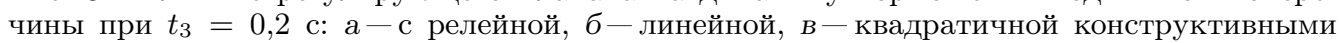
характеристиками

возвратный цилиндр», соответствующая скорости движения поперечины, успевает снизиться с $200 \mathrm{~mm} / \mathrm{c}$ до $148 \mathrm{~mm} / \mathrm{c}$.

При торможении клапаном с линейной конструктивной характеристикой (рис. 3, б) снижение скорости $V_{1}$ начинается гораздо раньше, чем в случае релейного клапана, а пиковое значение ускорения $j_{1}$ составляет $3 \mathrm{~m} / \mathrm{c}^{2}$. Скорость поперечины в момент закрытия клапана равна $140 \mathrm{~mm} / \mathrm{c}$.

Торможение квадратичным клапаном (рис. 3 , в) характеризуется снижением скорости $V_{1}$ с самого начала закрытия клапана, причём кривая приходит к своему нулевому значению с минимальной крутизной. Пиковое значение ускорения $j_{1}$ наблюдается на середине хода клапана и составляет $1,6 \mathrm{~m} / \mathrm{c}^{2}$. Поперечина замедляет свой ход до скорости $V_{2}$, равной 125 мм/с.

На рис. 4 приведены изменения

$V_{2}, \%$

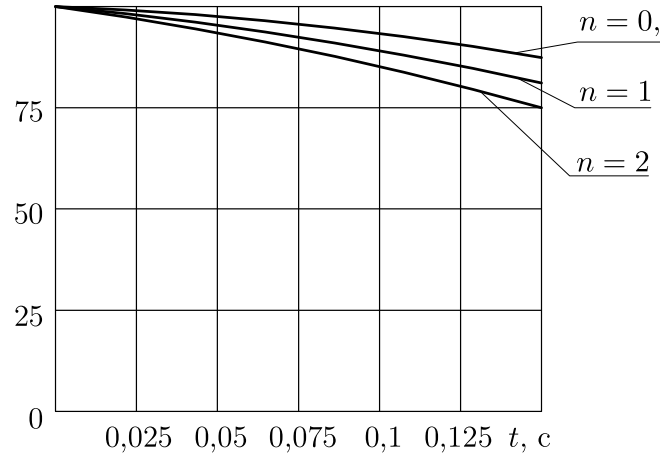

Рис. 4. Изменение скорости $V_{2}$ движения поперечины в зависимости от времени закрытия регулирующего клапана $t_{3}$ и вида его конструктивной характеристики $n$ скорости $V_{2}$ (в \%) движения поперечины от времени закрытия регулирующего клапана $t_{3}$ и вида его конструктивной характеристики $n$. Из графиков видно, что наибольшее снижение скорости $V_{2}$ (до $75 \%$ ) даёт клапан с квадратичной конструктивной характеристикой. Линейный клапан обеспечивает падение скорости до $78 \%$, а релейный - до $81 \%$.

Коэффициент качества гидросистемы $\alpha$, показывающий долю сопротивления регулирующего клапана в общем сопротивлении магистрали, оказывает существенное влияние на динамику процесса торможения подвижной поперечины. Причём, чем выше коэффициент $\alpha$, тем процесс торможения эффективнее. Идеальным является случай, когда $\alpha=1$, но в реальных системах гидравлических прессов это значение недостижимо. Достижимой величиной при 
рациональном проектировании гидросистем являются величины $\alpha$ в пределах $0,4 \div 0,6$.

Из графика (рис. 5) видно, что независимо от величины коэффициента $\alpha$ и вида конструктивной характеристики регулирующего клапана снижения скорости $V_{2}$ до нулевого значения не происходит. При этом наилучшую картину торможения дает клапан с квадратичной конструктивной характеристикой $(n=2)$, наихудшую - с релейной $(n=0,5)$.

Анализ математической модели первого этапа торможения подвижной поперечины на базе системы (1) показывает, что даже при самых благоприятных режимах торможения выбег поперечины вверх неизбежен. При этом процесс торможения

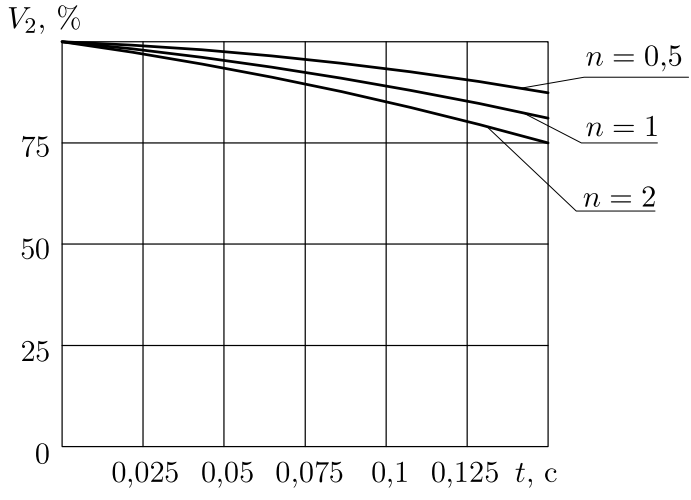

Рис. 5. Изменение скорости $V_{2}$ движения поперечины в зависимости от коэффициента $\alpha$ и вида конструктивной характеристики $n$ регулирующего клапана сопровождается гидроударными явлениями различной интенсивности. Увеличение времени торможения свыше 0,2 с позволяет избежать ударов, но добиться падения скорости $V_{2}$ до нуля за время закрытия клапана всё равно невозможно.

4. Анализ математической модели второго этапа торможения. Анализ уравнения (2) показывает, что выбег подвижной поперечины после закрытия регулирующего клапана является результатом сложного взаимодействия ряда сил: инерционных, гидростатических, трения. В зависимости от того, какие силы преобладают в каждом конкретном случае, поперечина совершает выбег той или иной величины. При этом выбег подвижной поперечины включает в себя две составляющие: инерционную, обусловленную уровнем накопленной подвижными частями кинетической энергии, и гидростатическую, обусловленную упругостью рабочей жидкости в наполнительно-сливном трубопроводе. А величина выбега после закрытия регулирующего клапана в значительной степени определяется квадратом скорости поперечины в момент его закрытия. Это подтверждается кривыми выбегов, приведёнными на рис. 6, после закрытия клапана с релейной (рис. 6, а), линейной (рис. 6, б) и квадратичной (рис. 6, в) конструктивными характеристиками. При $t_{3}=0,2$ с величина выбега составляет: при торможении релейным клапаном - 18 мм, линейным - 12 мм, квадратичным - 11 мм.

Заключение. Численным анализом математической модели торможения подвижной поперечины гидравлического пресса в верхнем положении установлено, что регулирующие свойства у впускного клапана возвратных цилиндров практически отсутствуют, из-за чего даже при самых благоприятных режимах торможения выбег поперечины вверх, приводящий к вакуумированию системы возвратных цилиндров, неизбежен. При этом торможение подвижной поперечины сопровождается гидроударами различной интенсивности независимо от вида конструктивной характеристики регулирующего клапана и времени его закрытия, а также величины коэффициента $\alpha$. 


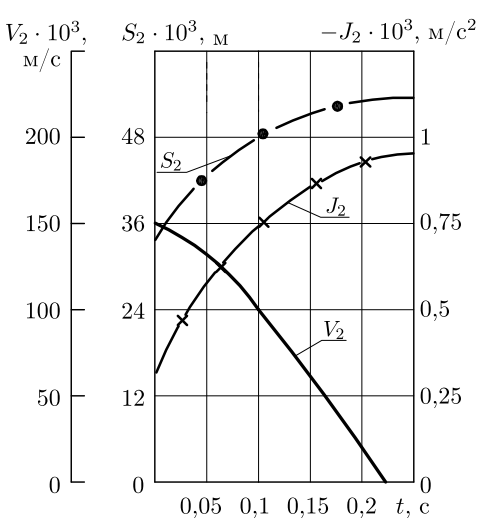

a

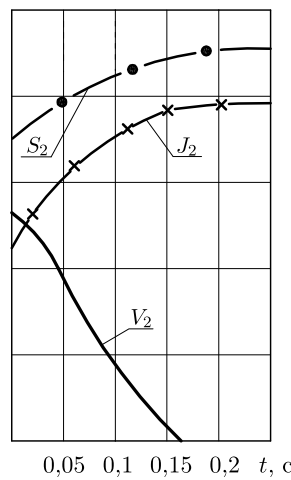

б

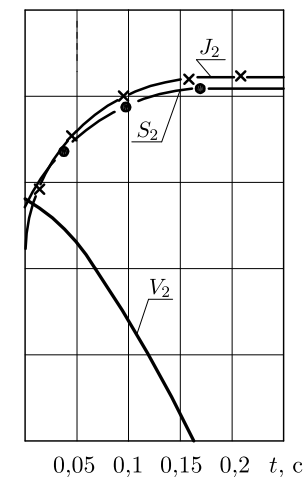

$B$

Рис. 6. Влияние регулирующего клапана на выбег подвижной поперечины при $t_{3}=0.2 \mathrm{c}$ : $a-c$ релейной, $\sigma$ - линейной, $в$ - квадратичной конструктивными характеристиками

Увеличение времени торможения свыше 0,2 с позволяет снизить удары, однако затяжное торможение приводит к снижению производительности пресса, что является недопустимым.

Анализ рассмотренной математической модели позволяет сделать вывод, что существующий способ торможения подвижной поперечины в верхнем положении является неэффективным. Для обеспечения быстрого и безударного торможения необходимо разработать новый механизм осуществления этого процесса.

\section{БИБЛИОГРАФИЧЕСКИЙ СПИСОК}

1. Левитский Н. И., Цуханова Е. А Расчёт управляющих устройств для торможения гидроприводов. - М.: Машиностроение, 1970. - 232 с.

2. Шинкаренко O.M., Корчак E. C. Торможение подвижных частей ковочных прессов с насосно-аккумуляторным приводом // Извест. ТулГУ. Серия. Механика деформируемого твёрдого тела и обработка металлов давлением, 2006. - № 1. - С. 346-355.

3. Соколов Л. Н., Золотухин В. Н., Ефимов В. Н. и др. Ковка слитков на прессах. - Киев.: Техника, 1984. - С. 108-124.

4. Белов А.Ф., Розанов Б. В., Лини, В. П. Объемная штамповка на гидравлических прессах / ред. А. Ф. Белов. - М.: Машиностроение, 1986. - 256 с.

Поступила в редакцию $27 / \mathrm{X} / 2008$;

в окончательном варианте - 24/VII/2009. 
MSC: 93A30

ANALYSIS OF MATHEMATICAL MODEL OF HYDRAULIC PRESS RAM BRAKING IN UPPER POSITION

\section{E. S. Korchak}

Donbass State Engineering Academy,

72, Shkadinova str., Kramatorsk, 84313, Ukraine.

E-mail: helen_korchak@ukr.net

Mathematical model of hydraulic press ram braking in upper position is considered. The influence of control valve closing time, its constructive characteristics and coefficient of hydraulic system quality on dynamics of ram braking while different stages is analyzed.

Key words: braking, ram, hydraulic press, control valve, constructive characteristics, closing time.

Original article submitted $27 / \mathrm{X} / 2008$; revision submitted 24/VII/2009.

$\overline{\text { Elena S. Korchak (Ph. D. (Techn.) }}$, Lecturer, Dept. of Machinery \& Metalforming Technology. 